\title{
Endoscopic treatment of maxillary inverted papilloma*
}

\author{
Fabio Pagella ${ }^{1}$, Georgios Giourgos ${ }^{1,2}$, Elina Matti ${ }^{1}$, Frank R. Canevari $^{1}$, \\ Paolo Carena ${ }^{1}$ \\ ${ }^{1}$ Department of Otorhinolaryngology, University of Pavia and Foundation IRCCS Policlinico S. Matteo, \\ Pavia, Italy \\ ${ }^{2} \mathrm{PhD}$ course in experimental surgery and microsurgery, University of Pavia, Italy
}

SUMMARY Objectives: Evaluate the efficacy of endoscopic treatment in maxillary inverted papilloma (IP).

Methodology: Between July 2002 - April 2008, 20 patients affected by maxillary localization of IP were treated in our Clinic. All patients underwent endoscopic treatment consisting of an endoscopic medial maxillectomy (simple or extended), or attachment-site endoscopic tumour surgery.

Results: The cohort was composed of 20 patients ( $0^{n}: 15$, $9: 5$ ), mean age 58 years, and included 21 endoscopic resections of maxillary IP. Minimum follow-up: 24 months, mean follow-up: 50 months. We registered only 1 case of tumour persistence/recurrence after 15 months, which underwent a second endoscopic treatment. No association with malignant lesions was noted. The efficacy of the endoscopic treatment was $95 \%$ after primary surgery, and 100\% after endoscopic revision.

Conclusions: Our experience demonstrates the efficacy of endoscopic treatment in maxillary $I P$. Based on its reduced morbidity in comparison to external approaches and its good control of the disease, we consider it our standard treatment for maxillary-originated inverted papilloma.

Key words: endoscopy, maxillary sinus, functional endoscopic sinus surgery, inverted papilloma, maxillectomy

\section{INTRODUCTION}

Inverted papilloma (IP), as is well described in the literature, although infrequent, is one of the most common epithelial tumours of the sinonasal area. It accounts for around $0.5 \%$ to $4 \%$ of all nasal neoplasias and presents a male predominance of 2-4:1 ${ }^{(1)}$. The average age at presentation is within the fifthsixth decades of life ${ }^{(2)}$. Historically, the most common sites of origin are the lateral nasal wall $(82 \%)$, maxillary sinus $(53.9 \%)$, ethmoid sinus $(31.6 \%)$, frontal sinus $(6.5 \%)$ and sphenoid sinus $(3.9 \%)^{(3)}$. The etiology is still unknown and a possible relationship with HPV (subtypes $6,11,16,18$ ) has been noted in almost $40 \%$ of cases, among other contributing factors ${ }^{(4)}$. Although a benign tumour, its possible local aggressiveness, recurrence rate and referred association with malignant lesions have been the crucial characteristics that have led to an aggressive treatment ${ }^{(5)}$.

Surgery emerged as the treatment of choice and, until the recent past, exeresis through external approaches (such as mid- facial degloving or lateral rhinotomy) has traditionally been considered the gold standard of therapy. However, in recent years, all the aforementioned tumour characteristics have been the focus of scientific work and debate, and have consequently been reconsidered. At the same time, endoscopic techniques have been advocated in the treatment of several diseases of the sinonasal tract, including benign and malignant neoplasias; as a result, several works have underlined the efficacy and superiority of the endoscopic vs. external approaches for most cases of IPs, confirmed by a recent metanalysis (surgical efficacy of endoscopic vs. external approaches, $88 \%$ vs. $80 \%)^{(5)}$. Such superiority has been revealed by various publications studying the endoscopic exeresis of sinonasal IPs from all nasal subsites ${ }^{(6-12)}$. To our knowledge, not many works specifically treat the maxillary-originated IP ${ }^{(13,14)}$; moreover, some debate still exists as to whether a pure endoscopic procedure, without an additional external approach, can guarantee an acceptable level of cure.

The aim of our work is to present our experience with the 
endoscopic treatment of maxillary IP; the reason for such analysis is that the maxillary sinus is the most frequently involved sinus in sinonasal IP. Moreover, we think that as the antrum resembles a 'one-entrance' rigid box, we may receive accurate information on the attachment site of the tumour, growing patterns and the effectiveness of several endoscopic approaches. Our results confirm the efficacy of the endoscopic approach that, we think, represents the gold standard treatment in most cases of maxillary IP.

\section{MATERIALS AND METHODS}

Study design

After approval by the Ethics Committee, a retrospective data review was performed on the patients that underwent endoscopic sinus surgery in our Clinic between 1995 and 2010; we registered 98 cases of sinonasal IP. The present work focuses on the last 20 consecutive maxillary IP patients, treated between July 2002 - April 2008 and having a follow-up $\geq 24$ months; our paper updates the results of a recent presentation and residency thesis (Corresponding author, personal communication ERS\&ISIAN 2008).

\section{Patient selection}

All patients underwent a thorough pre-operative endoscopic evaluation; by the time of the endoscopic biopsy both computed tomography (CT) and magnetic resonance imaging studies (MRI) had already been performed (Figures 1,2). Based on all clinical and radiological findings, the patients were retrospectively classified according to the staging systems reported by Krouse, Han et al. and Cannady et al. (Table 1) ${ }^{(3,15,16)}$.

\section{Surgical procedure}

All cases were treated by a pure endoscopic approach and the procedures were video-registered for subsequent review and analysis. In none of this recent series did we note an extranasal IP extension. All surgical acts were performed under general anaesthesia in a slightly reversed Trendelenburg position $\left(30^{\circ}\right)$. Cottonoids soaked in oxymetazoline solution were positioned in the nasal cavity for nasal decongestion. One percent lidocaine with 1:100 000 epinephrine was subsequently injected at the level of the root of the middle turbinate and the tumour itself. Surgical treatment followed the guidelines already published by our group ${ }^{(11)}$. An endoscopic medial maxillectomy, or an extended endoscopic medial maxillectomy (modified 'endonasal Denker operation') was applied to most cases ${ }^{(17)}$. In the former, resection of the medial maxillary wall extended from the posterior maxillary sinus wall to the Hasner valve. In the latter type of maxillectomy instead, resection was extended anteriorly to include the lacrimal pathway along with the anterior third of the inferior turbinate, so as to remove the entire medial maxillary wall; in doing so, the naso-lacrimal duct (NLD) was exposed and incised at its proximal end and its patency was confirmed by an external lacrimal-sac compression. To achieve control of the anterior portion of the maxillary sinus (anterolateral maxillary wall, alveolar recess, superomedial angle, anterior half of the superior wall) the piriform crest was drilled, thus performing a modified 'endonasal Denker operation' (Figure 3). In these two groups of patients a subperiosteal dissection plane was performed along all the exposed maxillary walls. A third group of patients was approached following the principles of the recent endoscopic tumour site-attachment surgery ${ }^{(18-21)}$. The main differentiation in this group is that the tumour mass was sequentially debrided along its pedicle and followed to its attachment site, but the subperiosteal dissection and consequent drilling was performed only at that site of attachment. A comparative analysis of those subgroups is beyond the scope of the present work, in which we would rather focus on the global success of the endoscopic maxillary IP treatment. In all cases we used microdebriders, either straight or curved, along with $0-45-70^{\circ}$ angled scopes; the tumoural mass was reduced by the debriders and then a subperiosteal resection was performed as previously stated. Subsequently, the underlying bone was drilled with straight and curved drills. No navigation systems were used during the procedures. Both the resected and debrided tumoural masses (by suction traps) were sent for histology. Finally, the nasal cavity was packed for 1 or 2 days. The patient received an ample-spectrum antibiotic therapy for some days and saline solution irrigations were recommended. In NLD resection cases, an ophthalmologist performed postoperative lavages of the residual lacrimal pathway, until a patent rhinostomy was endoscopically observed and epiphora was not noted. All patients were followed by endoscopic controls every 2 months during the first post-op year, every 4 months the second year, twice per year till the fifth year and then annually. MRI was performed post-operatively on all patients (at least 12 months after the procedure) and biopsies were taken in local anaesthesia from any suspicious cases.

\section{RESULTS}

From 1995, 98 patients were treated in our Clinic for sinonasal IP and form the historical group. We considered the cases treated after 2002 and reviewed 27 patients that received an endoscopic treatment for maxillary IP; 20 of them fulfilled the request of at least 24 months of follow-up. The group consisted of 15 males and 5 females, mean age 58 years (min $35 \mathrm{y}-\max 81 \mathrm{y}$ ), affected by a maxillary IP and treated with a pure endoscopic approach. Of those 20 patients, 7 (35\%) had already been treated in other institutions for sinusitis/polyposis with subsequent histological response of IP and then referred to our institution. The left side was more commonly involved than the right $(13 / 20,65 \%)$. The initial symptoms were unilateral nasal obstruction $(14 / 20,70 \%)$, facial pain-headache (4/20, $20 \%)$ and rhinorrhoea $(3 / 20,15 \%)$; interestingly, 2 patients $(2 / 20,10 \%)$ referred no nasal or other symptoms. All of them $(20 / 20,100 \%)$ received a pure endoscopic approach. The site of attachment was registered intra-operatively as: superior $(2 / 20)$, inferior $(5 / 20)$, posterior $(7 / 20)$, medial $(1 / 20)$, anterolateral $(3 / 20)$ and dubious (2/20) (Table 2). Type 2 resection was performed in 3 patients (15\%), type 3 in 7 cases $(35 \%)$ and attachment-site surgery in 10 of them $(50 \%)$. The mean followup was of 50 months ( $\min 24-\max 87)$. The mean hospitaliza- 


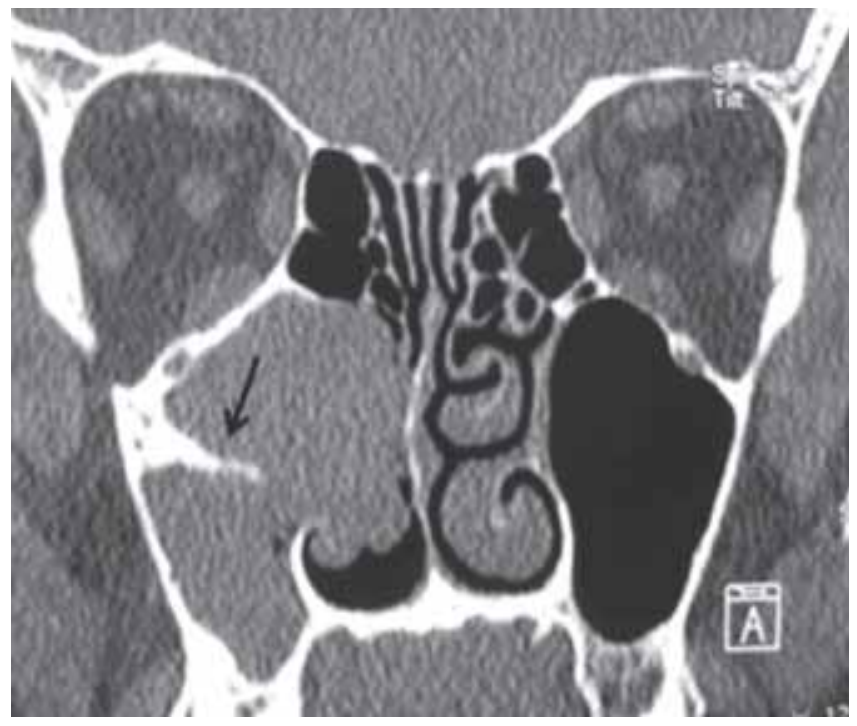

Figure 1. Computed Tomography. Coronal CT scan of an inverted papilloma of the right maxillary sinus. Observe the new bone formation and hyperostosis at the site of tumour attachment (black arrow).

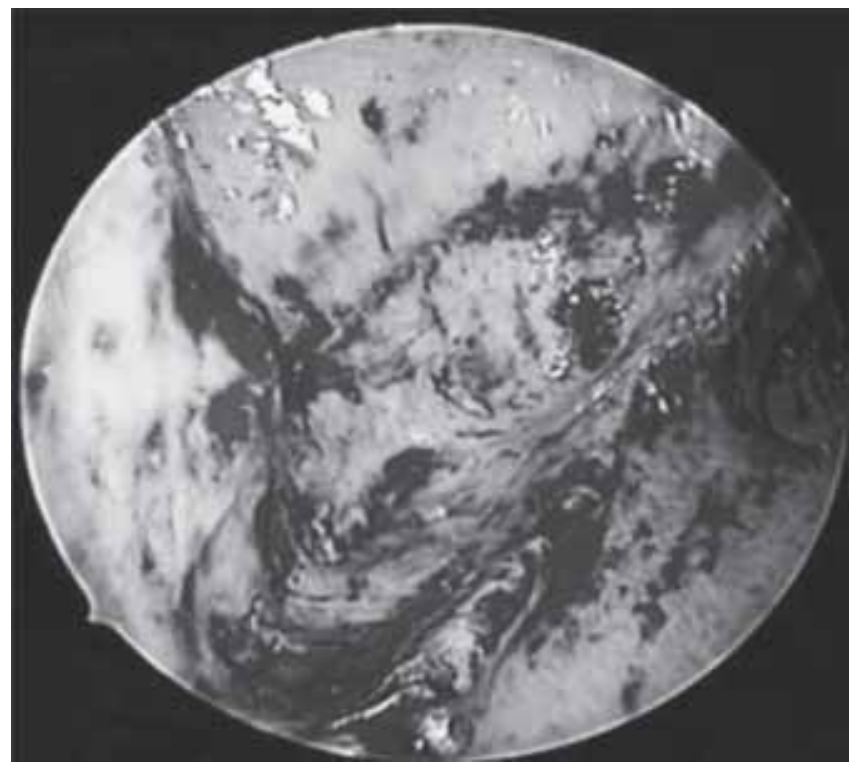

Figure 3. Extended medial maxillectomy. Endoscopic $0^{\circ}$ picture of a right extended medial maxillectomy at the end of the procedure.

tion rate was 2.4 days. No major complications occurred intraor post-operatively. One $(1 / 20,5 \%)$ patient presented with a post-op stenosis of the NLD and underwent a successful endoscopic dacriocystorhinostomy (DCR). In 3 patients $(15 \%)$ we registered a mild-moderate degree of facial paresthesia in the area innervated by the inferomedial branches of the V2 cranial nerve; all 3 cases had received an extended maxillectomy (with piriform crest drilling) and the symptom resolved gradually after some months. No case of synchronous/metachronous neoplasia was noted.

Fifteen months from the initial treatment, $1(1 / 20,5 \%)$ patient presented a persistence/recurrence of IP at the same site as the

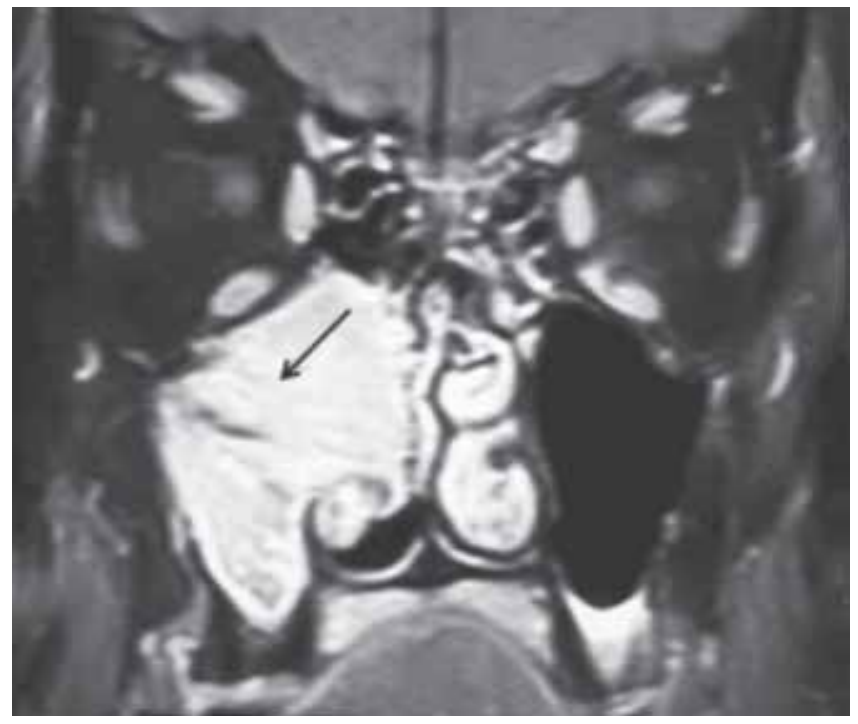

Figure 2. Magnetic resonance with contrast (gadolinium). T1 Coronal MRI scan of the previous case. Notice the convoluted cerebriform pattern pointing at the hyperostotic bone (black arrow).

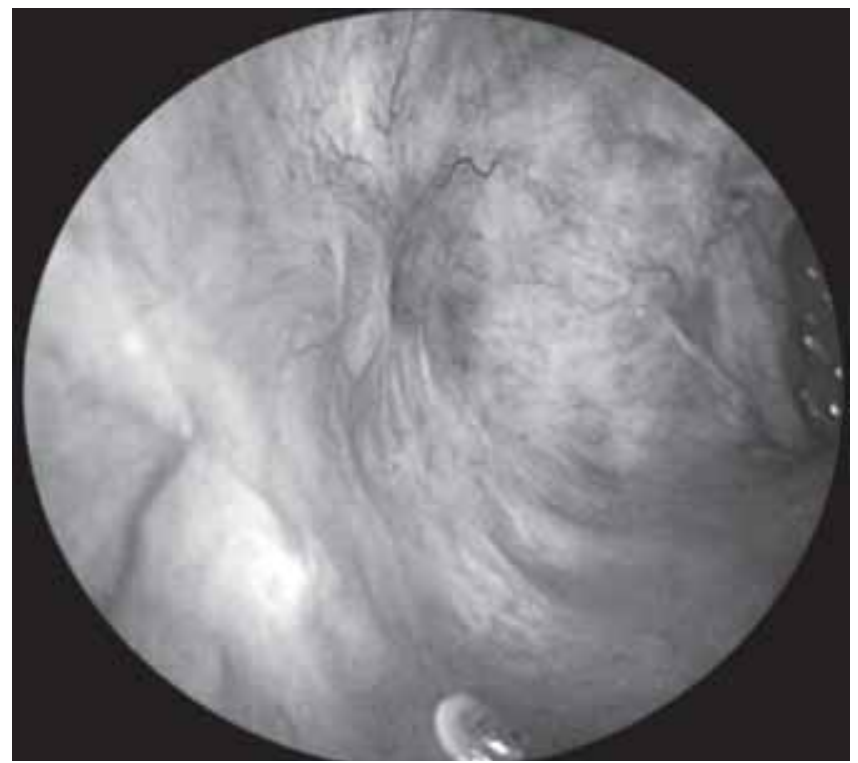

Figure 4. Endoscopic control of extended medial maxillectomy. Endoscopic $0^{\circ}$ picture of the previous (Figure 3 ) case at 2 years follow up. Observe the fibrosis and obliteration of the sinus.

initial procedure. This was studied through an MRI and then biopsied; he underwent an endoscopic revision surgery and to date remains free of disease. Within our group, the efficacy of the primary endoscopic approach to maxillary IP is $95 \%$ (19/20); after revision endoscopic surgery, it reaches $100 \%$ (20/20).

\section{DISCUSSION}

Schneiderian inverted papilloma (IP) is one of the most common epithelial tumours of the sinonasal area. It accounts for $0.5 \%$ to $4 \%$ of all nasal tumours and, to present, its aetiology remains unknown ${ }^{(1)}$. Historically, the maxillary sinus was involved in around $54 \%$ of cases ${ }^{(3)}$. However, according to 
Table 1. Classification according to Krouse, Han et al., Cannady et al. Staging Systems.

\begin{tabular}{lcccc}
\hline \multirow{3}{*}{ Krouse } & $\underline{\mathrm{T} 1}$ & $\underline{\mathrm{T} 2}$ & $\underline{\mathrm{T} 3}$ & $\underline{\mathrm{T} 4}$ \\
& 0 & 5 & 15 & 0 \\
\multirow{2}{*}{ Han } & $\underline{\text { Group 1 }}$ & $\underline{\text { Group 2 }}$ & $\underline{\text { Group 3 }}$ & $\underline{\text { Group 4 }}$ \\
& 5 & 15 & 0 & 0 \\
\multirow{2}{*}{ Cannady } & $\underline{\mathrm{A}}$ & $\underline{\mathrm{B}}$ & $\underline{\mathrm{C}}$ & \\
\hline
\end{tabular}

Table 2. Intra-operative research of the site of tumour attachment.

\begin{tabular}{lccccc} 
& Superior & Inferior & Posterior & Medial & Anterolateral \\
Cohort & 2 & 5 & 7 & 1 & 3 \\
Percentage & $10 \%$ & $25 \%$ & $35 \%$ & $5 \%$ & $15 \%$ \\
\hline
\end{tabular}

recent publications, where the site of tumour attachment was researched, a pure maxillary sinus' involvement represents the $31-39 \%{ }^{(20-22)}$. Its presentation is usually that of a unilateral polypoid mass with multiple digitations involving primarily the middle meatus. Usually both CT and MRI are performed, with the former offering information on the bony framework anatomy and chronic osteitis/neo-osteogenesis signs, the latter revealing the true neoplastic extension (vs. inflammatory changes), the 'convoluted cerebriform' radiological pattern and other indirect signs of the possible site of attachment ${ }^{(23-25)}$.

Historically, the gold-standard treatment of IPs has been external surgery, mostly through a midfacial degloving or a lateral rhinotomy approach ${ }^{(5)}$. In the ' 90 s, the first studies of a pure endoscopic approach to IP revealed the possible advantages of such technique vs. open surgery ${ }^{(6,26)}$. In recent years, after several publications regarding the endoscopic resection of the sinonasal IP, a metanalysis underlined the major efficacy of the latter approach (12\% vs $20 \%$, endoscopic vs non-endoscopic recurrence rates) ${ }^{(5)}$. In the same year, a review cited $12 \%$ vs $17 \%$ recurrence rates of endoscopic vs external surgery ${ }^{(27)}$. Very recently another review of pure endoscopic series found a $9.3 \%$ recurrence rate for the contemporary cohort vs. $16.5 \%$ for the historical group ${ }^{(28)}$; this last paper, although biased, gives as an idea of the evolution of endoscopic techniques and the consecutive improvement in surgical results.

In the early ' 90 s, we introduced endoscopic sinus surgery into our armamentarium, treating initially inflammatory, and then benign and malignant sinus diseases. In 2004, we published our results (combined with the ORL department of Brescia) in treating 47 cases of IP with a $0 \%$ recurrence at a mean 55 months of follow-up. Since then, the basic surgical principles such as tumour debulking, subperiosteal dissection plane and underlying bone drilling continue to guide our procedures. For tumours involving the medial, posterior and superior (its posterior half) walls of the maxillary sinus, an endoscopic medial maxillectomy is usually the treatment of choice similar to Kamel's description ${ }^{29)}$. However, the anterior part of the maxillary sinus [anterolateral and superior (its anterior half) walls, inferomedial (alveolar recess) and superomedial angles], is the most difficult to unveil and consequently treat; thus, the extended endoscopic medial maxillectomy permits a safe endoscopic approach to the latter subsites. This approach is basically a modified (less-invasive) version of the 'endonasal Denker operation', so-called by Sturmann and Canfield; the same operation was performed under microscopic view in recent years by Draf ${ }^{(30-32)}$. In all our 8 cases involving the latter group of subsites ( 5 inferior, 3 anterolateral) we managed to reach and treat the tumour site by this extended approach. We did not perform the maxillary trephine procedure or any anterior antrostomies/antrotomies as proposed by various authors ${ }^{(33,34)}$; our opinion is that violation of the external bony framework may permit neoplastic exteriorisation within the facial soft tissues, as previously observed by our group, in particular when the anterolateral wall is involved. A possible alternative to such "difficult" subsites could be the trans-septal technique in which an anterior septal window is created, so as to permit access to the tumour attachment through the opposite nostril (35).

Regarding the treatment of the site of IP-attachment we ought to say that in recent years, thanks to curved debriders and drills (and, of course, angled scopes), a less-invasive endoscopic approach has been applied to sinonasal IPs; this technique is based on tumour pedicle research and consequent tumour-attachment site surgery and concentrates the subperiosteal dissection and bone drilling right at the site of tumour attachment. As some recent papers state, the identification of such site of attachment facilitates the resection with minimal morbidity ${ }^{(20,21)}$.

Following all these surgical principles, we declare a 95\% efficacy of the endoscopic approach to maxillary IP (100\% after revision endoscopic surgery). We have registered only one case of persistent/recurrent disease and we have reviewed the 
video of the procedure. In such a case we would rather refer to 'persistence of disease' than 'recurrence of disease', as the interested nasal subsite was the same as the primary lesion. The tumoural regrowth involved the superomedial angle of the maxillary sinus, just behind the NLD. Revision endoscopic surgery was successful and the patient results free of disease. Even if open approaches are still frequently used in revision surgery, we think that endoscopic treatment of recurrence/persistence of disease is possible in many cases of maxillary sinus IP, as long as the tumour has grown within the bony framework ${ }^{(34)}$. However, in cases of exteriorization of the tumour within the facial tissues we believe that a combined approach would be the most appropriate. In doubtful cases strict endoscopic control, radiological evaluation (MRI) and histological confirmation can keep the possible residual disease under control and permit a safe endoscopic revision treatment. One more aspect we would rather stress is the complete absence of malignancy association in our cohort, and, furthermore, to date, in our entire group of sinonasal IPs. At the same time we did not register any major complications during surgery or post-operatively. We encountered one case of NLD stenosis that was successfully treated by an endoscopic DCR some months after initial surgery.

Regarding follow-up, we performed a strict endoscopic postoperative control in all our patients. In cases where the entire maxillary mucosa was removed, we observed a massive fibrosis, which points to the obliteration of the sinus itself after around 1 year (Figure 4); this healing process should be kept on mind and not erroneously be seen as a residual tumour. However, in all cases and after at least 12 months from primary surgery, we performed an MRI and eventually an endoscopic biopsy if any doubts arose. Most authors concur that recurrent/residual disease usually presents within 24 months, which is why we believe that, for safe conclusions, studies should include patients with at least 24 months of follow-up ${ }^{(36)}$.

\section{CONCLUSION}

Our experience, though numerically limited, demonstrates the efficacy of the endoscopic treatment of maxillary inverted papillomas. The oncological efficacy of the technique at 50 months of mean follow-up reaches $95 \%$ after primary surgery $(100 \%$ after endoscopic revision surgery). No external approaches were performed, nor were major complications noted in this cohort. With the adjunct of curved drills and debriders under angled endoscopic guidance, most cases can be treated entirely through an endoscopic approach. In particular, treatment of the anterior part of the maxillary sinus IPs can be successfully addressed without external adjuncts. At the same time, revision surgery for persistence/recurrence can be performed in uncomplicated cases under endoscopic guidance. However, in the event of tumour exteriorization within the facial tissues, a combined approach is advised. In the era of mini-invasive surgical approaches and attachment-site endoscopic surgery, we would welcome comparative studies between the classic endoscopic maxillectomies and attachment-site endoscopic surgery approaches.

\section{AUTHORSHIP CONTRIBUTION}

All authors contributed to the database review, along with writing and editing of the paper.

\section{CONFLICT OF INTEREST}

The authors state that no conflicts of interests exist.

\section{REFERENCES}

1. Hyams VJ. Papillomas of the nasal cavity and paranasal sinuses. A clinicopathological study of 315 cases. Ann Otol Rhinol Laryngol. 1971; 80: 192-206.

2. Outzen KE, Grontveld A, Jorgensen K, et al. Inverted papilloma: incidence and late results of surgical treatment. Rhinology. 1996; 34: 114-118.

3. Krouse JH. Endoscopic treatment of inverted papilloma: safety and efficacy. Am J Otolaryngol. 2001; 22: 87-99.

4. Kim JY, Yoon JK, Citardi MJ, Batra PS, Roh HJ. The prevalence of human papilloma virus infection in sinonasal inverted papilloma specimens classified by histological grade. Am J Rhinol. 2007; 21: 664-669.

5. Busquets JM, Hwang PH. Endoscopic resection of sinonasal inverted papilloma: a meta-analysis. Otolaryngol Head Neck Surg. 2006; 134: 476-482.

6. Waitz G, Wigand ME. Results of endoscopic sinus surgery for the treatment of inverted papillomas. Laryngoscope. 1992; 102: 917-922.

7. Lund VJ. Optimum management of inverted papilloma. J Laryngol Otol. 2000; 114: 194-197.

8. Kuhn UM, Mann WJ, Amedee RG. Endonasal approach for nasal and paranasal sinus tumour removal. ORL J Otorhinol Relat Spec. 2001; 63: 366-371.

9. Wormald PJ, Ooi E, van Hasselt CA, Nair S. Endoscopic removal of sinonasal inverted papilloma including endoscopic medial maxillectomy. Laryngoscope. 2003; 113: 867-873.

10. Kaza S, Capasso R, Casiano RR. Endoscopic resection of inverted papilloma. Am J Rhinol. 2003; 17: 185-190.

11. Tomenzoli D, Castelnuovo P, Pagella F, et al. Different endoscopic surgical strategies in the management of inverted papilloma of the sinonasal tract: experience with 47 patients. Laryngoscope. 2004; 114: 198-200.

12. Pasquini E, Sciarretta V, Farneti G, Modugno GC, Ceroni AR. Inverted papilloma: a review of 89 cases. Am J Otolaryngol. 2004; 25: 178-185

13. Sadeghi N, Al-Dhahri S, Manoukian JJ. Transnasal endoscopic medial maxillectomy for inverting papilloma. Laryngoscope. 2003; 113: 749-753.

14. Jurado-Ramos A, Gutierrez Jodas J, Ropero Romero F, et al. Endoscopic medial maxillectomy as a procedure of choice to treat inverted papillomas. Acta Otolaryngol. 2009; 129: 1018-1025.

15. Han JK, Smith TL, Loehrl T, Toohill RJ, Smith MM. An evolution in the management of sinonasal inverting papilloma. Laryngoscope 2001; 111: 1395-1400.

16. Cannady SB, Batra PS, Sautter NB, Roh HJ, Citardi MJ. New staging system for inverted papilloma in the endoscopic era. Laryngoscope. 2007; 117: 1283-1287.

17. Brors D, Draf W. The treatment of inverted papilloma. Curr Opin Otolaryngol Head Neck Surg. 1999; 7: 33-38.

18. Tufano RP, Thaler ER, Lanza DC, Goldberg AN, Kennedy DW. Endoscopic management of inverted papilloma. Am J Rhinol. 1999; 13: 423-426

19. Lee TJ, Huang SF, Huang CC. Tailored endoscopic surgery for the treatment of sinonasal inverted papilloma. Head Neck. 2004; 26: 145-153.

20. Landsberg R, Cavel O, Segev Y, Khafif A, Fliss DM. Attachmentoriented endoscopic surgical strategy for sinonasal inverted papilloma. Am J Rhinol. 2008; 22: 629-634.

21. Woodworth BA, Bhargave GA, Palmer JN, et al. Clinical outcomes of endoscopic and endoscopic-assisted resection of inverted papillomas: A 15-year experience. Am J Rhinol. 2007; 21: 591-600. 
22. Sham CL, Woo JK, van Hasselt CA, Tong MC. Treatment results of sinonasal inverted papilloma: an 18-year study. Am J Rhinol Allergy. 2009; 23: 203-211

23. Yousuf K, Wright ED. Site of attachment of inverted papilloma predicted by CT findings of osteitis. Am J Rhinol. 2007; 21: 32-36.

24. Maroldi R, Farina D, Palvarini L, Lombardi D, Tomenzoli D, Nicolai P. Magnetic resonance imaging finding of inverted papilloma: differential diagnosis with malignant sinonasal tumours. Am J Rhinol. 2004; 18: 305-310.

25. Bhalla RK, Wright ED. Predicting the site of attachment of sinonasal inverted papilloma. Rhinology. 2009; 47: 345-348.

26. Stankiewicz JA, Girgis SJ. Endoscopic surgical treatment of nasal and paranasal sinus inverted papilloma. Otolaryngol Head Neck Surg. 1993; 109: 988-995.

27. Karkos PD, Fyrmpas G, Carrie SC, Swift AC. Endoscopic versus open surgical interventions for inverted nasal papilloma: a systematic review. Clin Otolaryngol. 2006; 31: 499-503.

28. Heathcote KJ, Nair SB. The impact of modern techniques on the recurrence rate of inverted papilloma treated by endonasal surgery. Rhinology. 2009; 47: 339-344

29. Kamel Rh. Transnasal endoscopic medial maxillectomy in inverted papilloma. Laryngoscope. 1995; 105: 847-853.

30. Denker A. Ein neuer Weg fur die Operation der malignen Nasentumoren. Munch Med Wochenschr. 1906; 53: 953-956.

31. Sturmann D. Die intranasale Eröffnung der Kieferhöhle. Berl Klin Wochenschr. 1908; 27: 1273-1274.

32. Canfield RB: The submucous resection of the lateral nasal wall in chronic empyema of the antrum, ethmoid and sphenoid. JAMA. 1908; 51: 1136-1141.
33. Wormald PJ, Ooi E, van Hasselt CA, Nair S. Endoscopic removal of sinonasal inverted papilloma including endoscopic medial maxillectomy. Laryngoscope. 2003; 113: 867-873.

34. Philpott CM, Dharamsi A, Witheford M, Javer AR. Endoscopic management of inverted papillomas: long-term results - the St. Paul's Sinus Centre experience. Rhinology. 2010; 48: 358-363.

35. Harvey RJ, Sheehan PO, Debnath NI, Schlosser RJ. Transseptal approach for extended endoscopic resections of the maxilla and infratemporal fossa. Am J Rhinol Allergy. 2009; 23: 426-432.

36. Lawson W, Patel ZM. The evolution of management for inverted papilloma: an analysis of 200 cases. Otolaryngol Head Neck Surg. 2009; 140: 330-335.

\section{Georgios Giourgos, MD}

Department of Otorhinolaryngology

University of Pavia and Foundation IRCCS Policlinico S.

Matteo

V. le Golgi 19

27100 Pavia

Italy

Tel: +39-38-250 3350

Fax: +39-38-252 8184

E-mail: ggdoc1@yahoo.com 